\title{
Daniel N. Stern: una biografia intersoggettiva ${ }^{1}$
}

\author{
di Aluette Merenda* e Alessandra Salerno**
}

\begin{abstract}
Il presente contributo rappresenta una nota sulla biografia di Daniel Stern ove emerge una prospettiva intersoggettiva che attraversa la sua vita come studioso delle relazioni primarie. In particolare, viene enfatizzato il paradigma del changing di tutte le forme vitali (arte, sviluppo, psicologia e psicoterapia) che lo porta a comparare il contesto dello sviluppo infantile con l'ambito del setting terapeutico psicoanalitico, riattualizzando i medesimi costrutti e schemi relazionali impliciti, individuati nello sviluppo della relazione tra bambino e caregiver.
\end{abstract}

Parole chiave: intersoggettività, changing, relazioni primarie, forme vitali, psicoterapia, sviluppo.

\section{Daniel N. Stern: an intersubjective biography}

This paper represents a note on Daniel Stern's biography in which an intersubjective perspective emerges through his life as academic as well as researcher of the primary relationships. In particular, its aim is to focus the changing paradigm of all forms of vitality (arts, development, psychology and psychotherapy) that leads him to compare the context of child development with the psychoanalytic process setting, updating the same constructs and relational implicit schemas, identified in the development of the relationship between child and caregiver.

Key words: intersubjectivity, changing, primary relationships, forms of vitality, psychotherapy, development.

Daniel N. Stern nasce il 16 Agosto 1934 a New York. Muore il 12 Novembre del 2012 a Ginevra. Laureatosi in Psicologia presso l'Harvard University (1956) e specializzatosi in Psichiatria all'Albert Einstein College (1960), prosegue il suo training iniziando una serie di proficue collaborazioni con il Bellevue Hospital (1962), il National Institute of Health (1964), l'ospedale Presbiteriano (1967) di New York ed il Columbia University Center for Psychoanalysis (1972). Professore aggiunto del dipartimento di Psichiatria della Cornell University Medical School (New York Hospital), professore onorario della Facoltà di Psicologia, Università di Ginevra. Più recentemente gli viene conferita la laurea ad honorem presso le Università italiane di Palermo

\footnotetext{
1 Il presente contributo rappresenta un'elaborazione aggiornata della prolusione presentata in occasione del conferimento al Prof. Daniel N. Stern della laurea ad honorem in Psicologia clinica dello sviluppo, il 13 Maggio 2006 (Palermo, Palazzo Steri), proposta dalla prof.ssa Angela Maria Di Vita, professore ordinario di Psicodinamica dello sviluppo e delle relazioni familiari della Facoltà di Scienze della Formazione, dell’Università degli Studi di Palermo.
} 
(2006) e Padova (2007), e consegue il titolo di dottorato onorario anche presso le Università di Copenhagen (2005), Mons Hainaut, Stoccolma (2009) e di Aalborg (2011).

Autore di diversi volumi, di cui la maggior parte tradotta in più di dieci lingue, se ne annoverano alcuni: The first relationship: infant and mother (Cambridge, MA: Harvard University Press, 1977; trad. it.: Le interazioni madre-bambino nello sviluppo e nella clinica, Milano: Raffaello Cortina, 1998); The interpersonal world of the infant: a view from psychoanalysis and developmental psychology, (New York: Basic Books, 1985; trad.it.: Il mondo interpersonale del bambino, Torino: Bollati Boringhieri, 1987); The Journal of a baby (New York: Basic Books, 1990; trad.it.: Diario di un bambino, Milano: Oscar Mondadori,1999); The motherhood constellation: a unifying view of parent-infant psychotherapies (New York: Basic Books, 1995, trad.it.: La costellazione materna: il trattamento psicoterapeutico della coppia madre-bambino, Torino: Bollati Boringhieri, 1995); The birth of a mother (scritto con Nadia Bruschweiler-Stern, New York: Basic Books, 1997, trad.it.: Nascita di una madre, Milano: Oscar Mondadori, 2000); The present moment in psychotherapy and everyday life, (New York: Norton and Odile Jacob, 2003; trad. it.: Il momento presente in psicoterapia e nella vita quotidiana, Milano: Raffaello Cortina, 2005); Forms of vitality: exploring dynamic experience in Psychology, the Arts, Psychotherapy and Development (New York: Oxford University Press, 2010; trad. it.: Le forme vitali. L'esperienza dinamica in psicologia, nell'arte, in psicoterapia e nello sviluppo, Milano: Raffaello Cortina, 2011) e l'ultimo: Change in psychotherapy: a unifying paradigm (New York: Norton W. Norton \& Company, 2010), realizzato dal The Boston Change Process Study Group ${ }^{2}$, di cui è stato membro attivo.

Impegnato nel campo della ricerca e della pratica clinica nell'ambito dell'osservazione del comportamento infantile, avvia la sua prima osservazione negli anni'50 inspirandosi al lavoro sui cuccioli di gatto di Jay S. Rosenblatt presso l'American Museum of Natural History (NY). Dall'osservazione del loro comportamento - i gattini fin dal primo giorno di vita si dirigevano verso il capezzolo della propria madre - Stern inizia a volere dimostrare quanto sia precoce e naturale 1'apprendimento della funzione interattiva tra genitori e figli, lavorando alla interrelazione tra psicologia dello sviluppo e psicoterapia psicodinamica; osservazione-sperimentazione sull'infanzia e ricostruzione clinica delle esperienze precoci; prospettiva intersoggettiva ed intrapsichica.

\footnotetext{
${ }^{2}$ The Boston Change Process Study Group (1995) nasce con l'arduo compito di comprendere una psicoanalisi che riconosca i contributi della teoria clinica, il conflitto e le motivazioni inconsce, le interazioni relazionali, le osservazioni sui modelli di sviluppo. Attraverso i suoi membri il gruppo porta in sé la conoscenza dei modelli dell'infant research e dell'esperienza della pratica psicoanalitica con lo sforzo di studiare il processo di cambiamento che avviene sia nello sviluppo, sia nella pratica clinica.
} 
Tra i più conosciuti esponenti della infant researh ha approfondito, utilizzando metodi osservativi e sperimentali, lo sviluppo del bambino nei primi anni di vita in riferimento alle sue competenze e agli stili interattivi, alla esplorazione della vita intrapsichica e alle modalità di costruzione del senso di Sè. Analizzando "micro-analiticamente" i gesti impliciti connessi agli stili comunicativi tra genitori e figli, ha individuato modalità di “dialogo interattivo",configurato dall'apporto attivo di ciascun polo della relazione.

I risultati delle sue ricerche, configurabili entro un framework teorico che si concettualizza dall'elaborazione e dall'incrocio di differenti costrutti mutuati dalla psicoanalisi, il cognitivismo ed il comportamentismo, le teorie sistemiche, la psicologia sociale e l'etologia, portano un contributo profondamente innovativo alle teorie dello sviluppo infantile. Privilegiando una prospettiva di studio intersoggettiva, l'assunto base di Daniel Stern configura lo sviluppo infantile entro un contesto relazionale ove il bambino e il proprio caregiver primario si orientano, fin dai primi momenti interattivi, mediante uno scambio emotivo-affettivo reciproco, nonché costante sul piano fisico e psicologico; entro tale prospettiva, avvia una sfida alle teorie sullo sviluppo infantile e sugli schemi evolutivi, per la scelta di un approccio normativo, piuttosto che patomorfico, prospettico piuttosto che retrospettivo.

Focalizzandosi sul processo di riorganizzazione delle prospettive soggettive sul Sé e sull'altro, egli delinea la progressiva maturazione dell'individuo rispetto ad un modello che si struttura lungo quattro differenti tipologie e sensi del Sé (emergente, nucleare, soggettivo, narrativo), al cui interno ogni esperienza nasce in un preciso momento evolutivo; le sue scoperte sui cambiamenti e sui passaggi evolutivi avvengono attraversando gli stadi dello sviluppo del Sé e si configurano entro un moving along, ovvero, un movimento evolutivo progressivo che comprende avvicinamenti, frustrazioni, adattamenti e cambiamenti, che conduce al processo d'individuazione psichica.

Daniel Stern concentra la sua osservazione scientifica particolarmente sugli aspetti del mondo rappresentazionale, piuttosto che sulle strutture mentali, non esperite e dunque slegate dall'esperienza; contrariamente ad altre precedenti visioni psicoanalitiche, per le quali il neonato è rappresentato simbioticamente con la figura di accudimento primario dalla quale gradualmente impara a riconoscersi per raggiungere un'adeguata autonomia, egli ritiene invece che già dalla nascita il bambino utilizzi come soggetto empatico le proprie capacità ed intenzionalità intersoggettive, finalizzate all'incontro con l'altro. In tale ottica, egli concettualizza gli "schemi dell'essere con", che si configurano nell'interazione del bambino con il caregiver e si organizzano entro un "modo di essere con" che fornisce la rappresentazione dell'esperienza interpersonale. Le osservazioni e le concettualizzazioni sulle rappresentazioni mentali, condivise successivamente con 
Nadia Bruschweiler, hanno rilevato le implicazioni psichiche rispetto alle particolari "costellazioni" materne (1999).

Il suo crescente interesse per la prospettiva intersoggettiva, lo porta a comparare il contesto dello sviluppo infantile con l'ambito del setting terapeutico psicoanalitico, riattualizzando i medesimi costrutti e schemi relazionali impliciti individuati nello sviluppo della relazione tra bambino e caregiver. Più particolarmente, individuando le specifiche differenziazioni contestuali, legate soprattutto alla diversità dei ruoli e delle finalità evolutive, secondo Daniel Stern i processi terapeutici emergenti possono essere letti attraverso lo stesso approccio evolutivo osservato per il periodo infantile. Al terapeuta l'adulto ripropone infatti inevitabilmente il modello di "conoscenza relazionale implicita", costruito nel corso del proprio ciclo vitale. A tale modello si integra successivamente il livello della "conoscenza procedurale della relazione" che, pur operando al di fuori dell'intenzionalità e dell'esperienza cosciente dell'individuo, inevitabilmente ne condiziona il comportamento.

Nella sua più attuale linea di ricerca, Daniel Stern, insieme al suo gruppo di lavoro (Boston Group for the Study of Change Process), osserva come risulti necessario al fine della terapia, "destrutturare" il paziente, aprirlo a nuove esperienze e vissuti per poterlo condurre verso nuove possibilità comportamentali e affettive. In questo senso, egli sottolinea la necessità di inserire il momento della spontaneità e dell'imprevisto come parte integrante della relazione terapeutica; questi momenti, denominati present moments e spesso lasciati ai margini nel trattamento terapeutico classico, acquisiscono invece per il professore Stern un valore terapeutico in their own right, divenendo momenti critici della seduta e promuovendo il cambiamento. I present moments, arricchiti dall'esperienza affettiva, scambio intersoggettivo e riconoscimento reciproco, consentono l'incontro autentico tra paziente e terapeuta.

Nelle sue numerose pubblicazioni, Daniel N. Stern ha costruito una prospettiva di studio sul mondo infantile, unificando aspetti di ricerca e aspetti di clinica, contestualizzando gli uni rispetto all'osservazione delle rappresentazioni mentali e delle costellazioni materne (1999) gli altri, nell'ambito del setting psicoterapeutico, riattualizzando i costrutti e gli schemi relazionali.

Negli ultimi trenta anni il suo lavoro di ricerca si è concentrato proprio sugli aspetti d'interconnessione tra la psicologia dello sviluppo infantile e la psicoterapia psicodinamica, tra l'osservazione infantile e la ricostruzione clinica delle esperienze interattive precoci, tra la prospettiva intrapsichica ed interpersonale, favorendo spunti di mediazione ed integrazione nella comprensione della teoria clinica, nella pratica e nello sviluppo.

Scegliamo di riportare una traccia della sua lectio magistralis, tenuta proprio presso la Facoltà di Scienze della Formazione di Palermo ove gli è stata conferita la laurea ad honoris causa, sul 
supporto della Fenomenologia:

« Ho sperato di trovare un dio o una dea dell'antichità che possedesse il dono della lettura della mente (non della divinazione) e che potesse offrirla ad un essere umano. Questo dono renderebbe le menti degli altri trasparenti. Devo ancora trovare una tale divinità. I miei colleghi, affidabili in questo campo, mi hanno assicurato che la mia ricerca è vana. Almeno nell'antichità occidentale la mente non era confinata né imprigionata nella testa o nel cuore di un individuo. La mente circolava più liberamente, ricevendo costantemente input dalla natura e dagli dei. Essa non apparteneva a qualcuno come proprietà privata e segreta. Non vi era una grande necessità del dono di rendere le menti altrui trasparenti.

Storicamente, nell'occidente moderno ed orientato scientificamente, noi abbiamo isolato la mente dal corpo, dalla natura e da altre menti. La nostra esperienza del corpo, della natura e delle altre menti deve essere costruita privatamente e forse piuttosto idiosincraticamente entro la nostra mente. Fino ai tempi più recenti, questa concezione è stato dominante ed ampiamente Indiscussa tranne che dai filosofi.

Adesso stiamo assistendo ad una rivoluzione. Non siamo tornati proprio alle concezioni dell'antichità, ma siamo piuttosto vicini ad esse. Questa rivoluzione è stata ispirata in larga misura dal lavoro del filosofo della fenomenologia Edmund Husserl, (1859-1938; 1960; 1962; 1964; 1980; 1989).

L'approccio fenomenologico è stato rivitalizzato dai filosofi contemporanei ed è stato incorporato da alcuni scienziati nelle attuali concezioni alternative sulla natura umana che stanno acquistando rilievo rapidamente (Clark, 1999; Damasio, 1999; Freeman, 1999; Gallagher, 1997; Marbach, 1996; Sheets Johnstone, 1999; Thompson, 2001; Varela, 1996; Zahavi, 1996). Secondo questa nuova concezione, la mente è incorporata e resa possibile dall'attività senso-motoria di un individuo. Essa è intrecciata con e con-creata dall'ambiente fisico immediatamente circostante. Essa è costituita attraverso le sue interazioni con altre menti. La mente assume e conserva la propria forma e la propria natura a partire da questo traffico continuo. La mente emerge ed esiste soltanto grazie alla continua interazione di processi cerebrali auto-organizzati con l'ambiente e con le altre menti. Senza queste interazioni costanti non vi sarebbe alcuna mente riconoscibile. Una delle conseguenze di questa visione fenomenologica della "cognizione incorporata" è che la mente sia, per sua natura, "intersoggettivamente aperta", dal momento che è costituita in parte mediante le sue interazioni con altre menti (Husserl, 1960; Zahavi,1996; 2001; Thompson, 2001). Ciò vuol dire che gli esseri umani possiedono un funzionamento mentale primitivo descritto come "l'esperienza passiva (non iniziata volontariamente) e pre-riflessa dell'altro in quanto essere incorporato uguale a se 
stesso"(Thompson, 2001, p.12).

Parlando in termini neurobiologici, si può notare come questa esperienza pre-riflessa dell'apertura intersoggettiva emerge da meccanismi quali i neuroni specchio, gli oscillatori adattivi ed altri processi simili, prossimi ad essere scoperti. Ma, a livello esperienziale, questa apertura intersoggettiva crea le condizioni per l'intersoggettività primaria (sincronia, imitazione, sintonizzazione, ecc.) riscontrata nella prima infanzia e per le manifestazioni dell'intersoggettività secondaria (come la "vera" empatia) riscontrata successivamente. Credo sia in questo senso che Stein Braten (1999) parli del bambino fatto dalla natura per incontrare gli "altri virtuali". Siamo pronti per entrare nella matrice intersoggettiva che è una condizione dell'essere umano.

Qualsiasi considerazione del processo di psicoterapia deve tener conto delle premesse precedenti. L'esistenza di una matrice intersoggettiva definisce il contesto psicologico nel quale la relazione terapeutica prende forma. Transfert e contro-transfert sono solo casi particolari di un medesimo processo. L’idea di una psicologia unidirezionale è impensabile in questa situazione».

Queste considerazioni gettano nuova luce sui momenti presenti. Gli incontri intersoggettivi sono di durata relativamente breve. Essi sono generati in uno o più momenti presenti. Pertanto, il momento presente rimane una fondamentale unità del processo di co-creazione della matrice intersoggettiva. Concludendo, la teoria intersoggettiva elaborata da Daniel N. Stern, riferita allo studio del "mondo interpersonale del bambino" (1985), ha costituito e ha individuato una innovativa configurazione dello sviluppo infantile, ove il bambino ed il proprio caregiver primario si orientano, fin dai primi momenti interattivi, mediante uno scambio emotivo-affettivo reciproco. Tale prospettiva, offrendo una valida alternativa ad altre concettualizzazioni sull'infant research, ha assunto particolare importanza per gli studiosi ma è diventata di fondamentale interesse per l'ampliarsi di ulteriori direzioni e gruppi di ricerca. Come approccio normativo e prospettico, la sua prospettiva di studio rappresenta uno strumento fondamentale per tutti coloro che interpretano il ruolo di docente, nei suoi tanti, vari e variati ruoli. Meriti scientifici, indubbio valore per la ricerca e per la didattica sono i tre elementi che emergono nell'attività di ricerca di Daniel N. Stern e nella sua figura di studioso e docente. Le sue ipotesi sono state avanzate e sviluppate nei minimi dettagli; sono state offerte con generosità alla comunità scientifica che le ha fatte proprie. La sue tematiche esprimono già una direzione di ricerca, teorica e clinica ma soprattutto un'apertura verso altri approcci psicoterapeutici che,con linguaggi diversi, sottolineano l'importanza di aspetti non interpretativi nella cura psicoterapica. 


\section{Riferimenti bibliografici}

Bråten S. (2009). The intersubjective mirror in infant learning and evolution of speech. Amsterdam/Philadelphia: John Benjamins Publishing Company 2009 (ISBN 97890227252128).

Clark H. H. (1999). Psycholinguistics. Cambridge(MA): MIT Encyclopedia of the Cognitive Sciences, MIT Press.

Damasio A. (1999). The feeling of what happens: body and emotion in the making of consciousness, New York: Harcourt.

Freeman T. C. A. (1999). Path perception and Filehne illusion compared: model and data. Vision Research, 39(16), 2659-2667 (ISBN 10.1016/S0042-6989(98)00293-4).

Gallagher S. A. (1997). Problem-based learning: Where did it come from, what does it do, and where is it going?. Journal for the Education of the Gifted, 20 (4), 332-362

Husserl E. (1960). Cartesian meditations: an introduction to Phenomenology. Nijhoff : The Hague.

Marbach E. (1996). Understanding the representational mind: a phenomenological perspective. Human Studies 19 (2):137-52.

Sheets Johnstone M. (1999). The primacy of movement. Amsterdam: John Benjamins (ISBN: 155619-194-4).

Stern D.N. (1977). The first relationship: infant and mother, Cambridge, MA: Harvard University Press (trad. it.: Le interazioni madre-bambino nello sviluppo e nella clinica, Milano: Raffaello Cortina, 1998).

- (1985). The interpersonal world of the infant: a view from psychoanalysis and developmental psychology, New York: Basic Books (trad.it.: Il mondo interpersonale del bambino, Torino: Bollati Boringhieri, 1987).

- (1990). The Journal of a baby, New York: Basic Books, 1990 (trad.it.: Diario di un bambino, Milano: Oscar Mondadori,1999).

- (1995). The motherhood constellation: a unifying view of parent-infant psychotherapies, New York: Basic Books (trad.it.: La costellazione materna: il trattamento psicoterapeutico della coppia madre-bambino, Torino: Bollati Boringhieri,1995).

- (2003). The present moment in psychotherapy and everyday life, New York: Norton and Odile Jacob (trad. it.: Il momento presente in psicoterapia e nella vita quotidiana, Milano: Raffaello Cortina, 2005).

The Boston Change Process Study Group (2010). Change in psychotherapy: a unifying paradigm, New York: Norton W. Norton \& Company.

Thompson E. (2001). Empathy and consciousness. Journal of Consciousness Studies, 8 (5-7), 1-32. 
Varela F.J. (1996). Neurophenomenology: a methodological remedy to the hard problem. Journal of Consciousness Studies. 3 (4), 330-50

Zahavi D. (1996). Edmund Husserl. Fænomenologi og kunst. Blaa Port ,37, 45-61. 